\title{
A Cooperation and Competition Model for Risk Management of a Two-Stage Supply Chain
}

\author{
Lina $\mathrm{Xu}^{1, *}$, Zhiqing Meng ${ }^{2}$ and Hongjian $\mathrm{Yu}^{3}$ \\ ${ }^{1}$ Keyi College, Zhejiang Sci-Tech University, Hangzhou, Zhejiang, China; \\ ${ }^{2}$ College of Economics and Management, Zhejiang University of Technology, Hangzhou, Zhejiang, China; \\ ${ }^{3}$ Alibaba Business School, Hangzhou Normal University, Hangzhou, Zhejiang, China \\ ${ }^{*}$ Corresponding author
}

\begin{abstract}
Based on the CVaR measure, this paper introduces a Cooperation and Competition model for the risk management of the two-stage supply chain. It is supposed that both the supplier and the retailer are risk-averse and their objectives are to minimize their own CVaR(Conditional Value-at-Risk) objective instead of the expected profit. We introduce an $\mathrm{e}^{*}-$ optimal equilibrium solution to this model, which implies the supplier and the retailer should give up a same value no more than $\mathrm{e}^{*}$ for their cooperation. It is proved that the $\mathrm{e}^{*}$-optimal equilibrium solution can be obtained by solving a corresponding mathematical programming problem. Numerical results show that this method is efficient to improve the risk management of the two-stage supply chain.
\end{abstract}

Keywords-conditional value-at-risk; equilibrium solution; supply chain management; risk management

\section{INTRODUCTION}

With the growing emphasis on globalization, supply chain management has been applied to many fields, such as production plan and financial management ${ }^{[1-2]}$. In order to alleviate the bad effects of supply chain disruptions that caused by various unpredictable risks, people introduced many risk control methods to improve the risk management of the supply chain $^{[3]}$. In 2000, the CVaR measure was introduced to solve the portfolio optimization problems and subsequently proved to be an efficient method for risk management ${ }^{[4-5]}$. Thus some researchers introduced the $\mathrm{CVaR}$ measure into the supply chain management and some interesting results are achieved ${ }^{[6-11]}$. Chen X. et al. ${ }^{[6]}$ proposed a model based on CVaR measure for the newsvendor model and obtained the optimal order quantity for the retailer with no shortage penalty to maximize his CVaR objective. Xu Minghui et al. ${ }^{[7]}$ extended the model proposed in [6] and obtained the optimal order quantity with shortage penalty for the retailer to maximize his CVaR objective. Gotoh and Takano ${ }^{[8]}$ provided analytical solutions and linear programming formulation of the minimization of CVaR in the newsvendor model. Cheng et al. ${ }^{[9]}$ introduced a bilevel programming model with the CVaR objective for the two-stage supply chain. Hsieh and Yu-Ting ${ }^{[10]}$ characterized the retailer' risk-embedded objective via CVaR measure and studied return policy of the manufacture and the optimal decision of the retailer. Caliskan-Demirag et al. ${ }^{[1]}$ analyzed a suppler's customer and retailer rebates in the context of modeling aversion by adopting CVaR measure. In general, most of the related literature assume that the supplier is risk-neutral and aims to maximize his expected profit or minimize his expected loss. However, many suppliers in the real world are risk-averse and want to reduce the potential risks, while such an issue is always been ignored.

In view of the above issue, this paper assumes that both the supplier and the retailer in the two-stage supply chain are riskaverse, and their objectives are to minimize their own CVaR objective. We propose a Cooperation and Competition model for the two-stage supply chain. In this model, the supplier decides the wholesale price while the retailer decides the order quantity. They compete with each other to minimize their own CVaR objective while they cooperate to get more profits. We introduce an $\mathrm{e}^{*}$-optimal equilibrium solution to this model, which implies the supplier and the retailer should accept the same concession no more than $e^{*}$ for their cooperation. It is proved that the $\mathrm{e}^{*}$-optimal equilibrium solution can be obtained by solving a corresponding mathematical programming problem. Then this model provides an efficient method to coordinate the decisions of the supplier and the retailer in a two-stage supply chain.

The rest of this paper is organized as follows. Section II introduces the notation in this paper and some preliminaries. Section III introduces the proposed model and its solutions. In Section IV, a numerical example is given to illustrate the performance of the proposed model, with conclusions given in Section V.

\section{ThE NotATION AND SOME PREPARING RESUlTS}

\section{A. The Notation}

Firstly, we introduce the notation as follows:

$x$ denotes the wholesale price of unit product

$b$ denotes the cost of unit product for the supplier

$a$ denotes the maximal wholesale price of unit product

$t$ denotes the excess penalty coefficient for the supplier $y$ denotes the retailer's order quantity

$r$ denotes the salvage price of unit product

$S$ denotes the shortage penalty price of unit product

$n$ denotes the retailer's minimal order quantity

$N$ denotes the retailer's maximal order quantity

$\xi$ denotes the stochastic price of the product

$\eta$ denotes the stochastic demand of the product

$\pi_{1}$ denotes the objective of the supplier

$\pi_{2}$ denotes the objective of the retailer. 


\section{B. VaR and CVaR}

Value-at-Risk (VaR) is a popular risk control measure which has achieved high status of being written into industry regulations. For a decision $x$, let $l(x, \xi)$ be the loss associated with the decision $x$ and the random variable $\xi$. For the confidence level $\alpha$, the $\alpha$-VaR with $x$ is defined as

$$
\operatorname{VaR}_{\alpha}(x)=\min \{y \in R \mid \operatorname{Pr}\{l(x, \xi) \leq c\} \geq \alpha\},
$$

where $\operatorname{Pr}\{l(x, \xi) \leq c\}$ denotes the probability of $l(x, \xi)$ below the value $c . \operatorname{VaR}_{\alpha}(x)$ represents the minimum loss of the decision maker under the confidence level $\alpha$. Artzner et al. ${ }^{[12]}$ and Mauser et al. ${ }^{[13]}$ pointed out that VaR has some undesirable mathematical characteristics such as nonsubadditivity and non-convexity, which always hinder its efficient usage in practice. Therefore Rockafellar and Uryasev $^{[4-5]}$ introduced another famous risk measure Conditional Value-at-Risk, which is defined as the expected value of loss above the $\operatorname{VaR}_{\alpha}(x)$. CVaR has some attractive properties such as coherence and convexity, which makes it is widely used in risk management. The $\mathrm{CVaR}$ with $X$ can be defined as

$$
\begin{aligned}
\operatorname{CVaR}_{\alpha}(x) & =E\left[l(x, \xi) \mid l(x, \xi) \geq \operatorname{VaR}_{\alpha}(x)\right] \\
& =\frac{1}{1-\alpha} \int_{l(x, z) \geq \operatorname{VaR}_{\alpha}(x)} l(x, z) f(z) d y,
\end{aligned}
$$

where $f(\cdot)$ is the probability density function of $\xi$ and $\operatorname{VaR}_{\alpha}(x)$ is defined by (1). Rockafellar and Uryasev [3] introduced the following auxiliary function

$$
F(x, \xi, u)=u+\frac{1}{1-\alpha} E\left[(l(x, \xi)-u)^{+}\right]
$$

and proved that the minimum of $C V a R$ can be obtained by minimizing this auxiliary function $F(x, \xi, u)$.

\section{THE PROPOSED MODEL}

For the two-stage supply chain consisting of a supplier and a retailer, we first give a description on the decision framework of the supplier and the retailer. For the supplier, we assume that he is risk-averse and his objective is to minimize the following CVaR objective

$$
\operatorname{CVaR}_{\alpha}(x)=\frac{1}{1-\alpha} \int_{L(x, y, z) \geq \operatorname{VaR}_{\alpha}(x)} L(x, y, z) \phi(z) d z
$$

where

$$
L(x, y, \xi)=y(\xi-x)^{+}+t y(x-\xi)^{+}
$$

is the loss of the supplier, $\phi(\cdot)$ is the density function of $\xi$ and $\alpha$ is the confidence level. Here, the first term in the right hand of (4) represents the loss of the supplier when he gives a low wholesale price, while the second does the loss when he gives a high wholesale price. There are the following constraints about the supplier's wholesale price $x . r<x, b<x, x<a$.

For the retailer, we also assume he is risk-averse and his objective is to minimize the following $C V a R$ objective

$$
\mathrm{CVaR}_{\beta}(y)=\frac{1}{1-\beta} \int_{l(x, y, z) \geq \operatorname{VaR}_{\beta}(y)} l(x, y, z) \varphi(z) d z,
$$

where $l(x, y, \eta)=(x-r)(y-\eta)^{+}+s(\eta-y)^{+}$

is the loss of the retailer, $\varphi(\cdot)$ is the density function of $\eta$ and $\beta$ is the confidence level. Here, the first term in the right hand of (5) represents the loss of the retailer for excess order, and the second does the shortage penalty for the lost sales of the retailer. There is the following constraint about the retailer's order quantity $y . n \leq y \leq N$. Then we introduce the Cooperation and Competition (CC) model as follows:

$$
\begin{aligned}
& (\mathrm{PM}) \min \pi_{1}(x, y)=\frac{1}{1-\alpha} \int_{L(x, y, z) \geq \operatorname{VaR}_{\alpha}(x)} L(x, y, z) \phi(z) d z \\
& \text { s.t. } r<x, b<x, x<a .
\end{aligned}
$$

$(\mathrm{PR}) \min \pi_{2}(x, y)=\frac{1}{1-\beta} \int_{l(x, y, z) \geq \operatorname{VaR}_{\beta}(y)} l(x, y, z) \varphi(z) d z$ s.t. $n \leq y \leq N$.

In order to solve this model, we introduce $f(x, y, u)$ and $g(x, y, v)$ as follows:

$$
\begin{aligned}
& f(x, y, u)=u+\frac{1}{1-\alpha} E\left[(L(x, y, \xi)-u)^{+}\right], \\
& g(x, y, v)=v+\frac{1}{1-\beta} E\left[(l(x, y, \eta)-v)^{+}\right] .
\end{aligned}
$$

With the result in II.B, the CC model is transformed into

$$
\begin{aligned}
& \text { (PM) } \min f(x, y, u)=u+\frac{1}{1-\alpha} E\left[(L(x, y, \xi)-u)^{+}\right] \\
& \text {s.t. } r<x, b<x, x<a, u \in R .
\end{aligned}
$$

$(\mathrm{PR}) \min g(x, y, v)=v+\frac{1}{1-\beta} E\left[(l(x, y, \eta)-v)^{+}\right]$

s.t. $n \leq y \leq N, v \in R$.

Now, we will introduce an optimal solution to the CC model and show how to obtain this optimal solution. For the problem (PM), let $\xi_{k}(k=1,2, \cdots, I)$ be the $k-t h$ value of the stochastic variable $\xi$. Then the problem (PM) can be transferred into the following mathematical programming:

$(\mathrm{PM}) \min f(x, y, u)=u+\frac{1}{1-\alpha} E\left[(L(x, y, \xi)-u)^{+}\right]$ 


$$
\begin{gathered}
=u+\frac{1}{(1-\alpha) I} \sum_{k=1}^{I} z_{1 k} \\
\text { s.t. } z_{1 k} \geq y w_{1 k}+t y w_{2 k}-u, z_{1 k} \geq 0, \\
w_{1 k} \geq \xi_{k}-x, w_{1 k} \geq 0, w_{2 k} \geq x-\xi_{k}, w_{2 k} \geq 0, \\
k=1,2, \cdots, I . r<x, b<x, x<a, u \in R .
\end{gathered}
$$

For the problem (PR), let $\eta_{k}(k=1,2, \cdots, J)$ be the $k$-th value of the stochastic variable $\eta$.Then the problem (PR) can be transferred into the following mathematical programming:

$$
\begin{gathered}
\text { (PR) } \min g(x, y, v)=v+\frac{1}{1-\alpha} E\left[(l(x, y, \eta)-v)^{+}\right] \\
=v+\frac{1}{(1-\alpha) J} \sum_{k=1}^{J} z_{2 k} \\
\text { s.t. } z_{2 k} \geq(x-r) w_{3 k}+s w_{4 k}-v, z_{2 k} \geq 0, \\
\quad w_{3 k} \geq y-\eta_{k}, w_{3 k} \geq 0, w_{4 k} \geq \eta_{k}-y, w_{4 k} \geq 0, \\
k=1,2, \cdots, J . \quad n \leq y \leq N, v \in R .
\end{gathered}
$$

Let

$X=\{(x, y, u, v) \mid r<x, b<x, x<p, n \leq y \leq N, u \in R, v \in R\}$ be the feasible set to the CC model. We introduce the following definition.

Definition (1). For any $(x, y, u, v) \in X$, if it satisfies $f\left(x_{f}^{*}, y_{f}^{*}, u_{f}^{*}, v_{f}^{*}\right) \leq f(x, y, u, v)$,

$g\left(x_{g}^{*}, y_{g}^{*}, u_{g}^{*}, v_{g}^{*}\right) \leq g(x, y, u, v),\left(x_{f}^{*}, y_{f}^{*}, u_{f}^{*}, v_{f}^{*}\right)$

is called an optimal joint solution of the supplier and $\left(x_{g}^{*}, y_{g}^{*}, u_{g}^{*}, v_{g}^{*}\right)$ is called an optimal joint solution of the retailer. Moreover, $f^{*}=f\left(x_{f}^{*}, y_{f}^{*}, u_{f}^{*}, v_{f}^{*}\right)$ is called the optimal joint value of the supplier and $g^{*}=g\left(x_{g}^{*}, y_{g}^{*}, u_{g}^{*}, v_{g}^{*}\right)$ is called the optimal joint value of the retailer.

Obviously, $\left(x_{f}^{*}, y_{f}^{*}, u_{f}^{*}, v_{f}^{*}\right)$ is an optimal decision of the supplier while $\left(x_{g}^{*}, y_{g}^{*}, u_{g}^{*}, v_{g}^{*}\right)$ is an optimal decision of the retailer. If $\left(x_{f}^{*}, y_{f}^{*}, u_{f}^{*}, v_{f}^{*}\right)$ equals to $\left(x_{g}^{*}, y_{g}^{*}, u_{g}^{*}, v_{g}^{*}\right)$, it is best for the supplier and the retailer to choose the same decisions. However, since the objectives of the supplier and the retailer are always conflict, $\left(x_{f}^{*}, y_{f}^{*}, u_{f}^{*}, v_{f}^{*}\right)$ is always different with $\left(x_{g}^{*}, y_{g}^{*}, u_{g}^{*}, v_{g}^{*}\right)$. Then we introduce the following definition.
Definition (2). Let $f^{*}$ be the optimal joint value of the supplier and $g^{*}$ be the optimal joint value of the retailer. For $e \geq 0$ and $(x, y, u, v) \in X \quad$, if it satisfies $f(x, y, u, v)-e \leq f^{*}, g(x, y, u, v)-e \leq g^{*},(x, y, u, v)$

is called an $e$-equilibrium solution of the supplier and retailer. $e$ is called an equilibrium value of the supplier and retailer.

The $e$-equilibrium solution provides the same concession value $e$ that the supplier and retailer need to give away for their cooperation. If $e=0$, the $e$-equilibrium solution turns out to be the optimal joint solution for the supplier and the retailer.

Lemma (1). Let $f^{*}$ be the optimal joint value of the supplier and $g^{*}$ be the optimal joint value of the retailer. For any $\left(x^{\prime}, y^{\prime}, u^{\prime}, v^{\prime}\right) \in X,\left(x^{\prime}, y^{\prime}, u^{\prime}, v^{\prime}\right)$ is an $e^{\prime}$-equilibrium solution for the supplier and the retailer, where $e^{\prime}=\max \left\{f\left(x^{\prime}, y^{\prime}, u^{\prime}, v^{\prime}\right)-f^{*}, g\left(x^{\prime}, y^{\prime}, u^{\prime}, v^{\prime}\right)-g^{*}\right\}$.

Proof. By the assumption, it follows that $f\left(x^{\prime}, y^{\prime}, u^{\prime}, v^{\prime}\right)-f^{*} \leq e^{\prime}, g\left(x^{\prime}, y^{\prime}, u^{\prime}, v^{\prime}\right)-g^{*} \leq e^{\prime}$,

which implies

$$
f\left(x^{\prime}, y^{\prime}, u^{\prime}, v^{\prime}\right)-e^{\prime} \leq f^{*}, g\left(x^{\prime}, y^{\prime}, u^{\prime}, v^{\prime}\right)-e^{\prime} \leq g^{*} \text {. }
$$

It follows with definition (2) that $\left(x^{\prime}, y^{\prime}, u^{\prime}, v^{\prime}\right)$ is an $e^{\prime}$ equilibrium solution of the supplier and the retailer. This completes the proof.

By Lemma III.A, there exists an equilibrium value $e$ for each point $(x, y, u, v)$ in $X$ such that $(x, y, u, v)$ becomes an $e$-equilibrium solution of the supplier and the retailer. In fact, we are interested in the minimum of all the equilibrium values.

Definition (3). Suppose that the set of all the equilibrium values of the supplier and retailer is denoted by $E$. If $e^{*}$ is the minimum element in $E$ and $\left(x^{*}, y^{*}, u^{*}, v^{*}\right)$ is an $e^{*}$ equilibrium solution of the supplier and the retailer, $\left(x^{*}, y^{*}, u^{*}, v^{*}\right)$ is called an $e^{*}$ - optimal equilibrium solution of the supplier and the retailer. $e^{*}$ is called an optimal equilibrium value of the supplier and the retailer.

The $e^{*}$-optimal equilibrium solution provides the minimum concession $e^{*}$ both the supplier and the retailer need to give away. It is fair for the cooperation between the supplier and the retailer and both the supplier and the retailer gives the minimum concession for their cooperation. In order to obtain the $e^{*}$-optimal equilibrium solution of the supplier and the retailer, we first give the following result. 
Theorem (1). Suppose that the feasible set $X$ is compact, and both $f(\cdot)$ and $g(\cdot)$ are continuous. There exists an $e^{*}$ optimal equilibrium solution of the supplier and the retailer.

Proof. By Lemma (1), it is obvious that $E \neq \Phi$. We will prove that $E$ is closed. Suppose that the sequence $\left\{e_{k}\right\} \in E$ satisfies $\lim _{k \rightarrow+\infty} e_{k}=e^{\prime}$ and $\left(x_{k}, y_{k}, u_{k}, v_{k}\right) \in X$ is an $e_{k}$ equilibrium solution of the supplier and the retailer. Since $X$ is compact, there exists a subsequence $\left\{\left(x_{k}^{1}, y_{k}^{1}, u_{k}^{1}, v_{k}^{1}\right)\right\}$ in the sequence $\left\{\left(x_{k}, y_{k}, u_{k}, v_{k}\right)\right\}$ such that $\left\{\left(x_{k}^{1}, y_{k}^{1}, u_{k}^{1}, v_{k}^{1}\right)\right\}$ is convergent. Without loss of generality, we assume that $\left(x_{k}, y_{k}, u_{k}, v_{k}\right) \rightarrow(c, x, u, v) \in X$, it follows with definition (2) that $f\left(x_{k}, y_{k}, u_{k}, v_{k}\right)-e_{k} \leq f^{*}$, $g\left(x_{k}, y_{k}, u_{k}, v_{k}\right)-e_{k} \leq g^{*}, k=1,2, \cdots$.

Since $f$ and $g$ are continuous, it follows with $k \rightarrow+\infty$ that $f(x, y, u, v)-e^{\prime} \leq f^{*}, g(x, y, u, v)-e^{\prime} \leq g^{*}$.

$(x, y, u, v) \in X$ is an $s^{\prime}$-equilibrium solution, and this proves tha $E$ is closed. Since it satisfies $e \geq 0$ for any $e \in E$, there exists the minimum element $e^{*}$ which becomes the optimal equilibrium value. The corresponding $e^{*}-$ equilibrium solution turns to be the $e^{*}$-optimal equilibrium solution of the supplier and the retailer. This completes the proof.

By Theorem (1), there always exists an $e^{*}$-optimal equilibrium solution of the supplier and the retailer. Let $X^{*}$ denotes the set of all the $e^{*}$-optimal equilibrium solutions of the supplier and the retailer. We have the following result.

Theorem (2). If $X, f$ and $g$ are convex, $X^{*}$ is convex. Proof. For any $z_{1}, z_{2} \in X^{*} \subset X$ and $\lambda \in(0,1)$, it follows with the convexity of $X$ that $z=\lambda z_{1}+(1-\lambda) z_{2} \in X$.We will prove that $z \in X^{*}$. Let $e^{*}$ be the optimal equilibrium value of the supplier and the retailer. Since $z_{1}, z_{2} \in X^{*}$, it follows $f\left(z_{1}\right)-e^{*} \leq f^{*}, g\left(z_{1}\right)-e^{*} \leq g^{*}$,

$$
f\left(z_{2}\right)-e^{*} \leq f^{*}, g\left(z_{2}\right)-e^{*} \leq g^{*},
$$

which implies $f\left(z_{1}\right) \leq f^{*}+e^{*}, g\left(z_{1}\right) \leq g^{*}+e^{*}$,

$$
f\left(z_{2}\right) \leq f^{*}+e^{*}, g\left(z_{2}\right) \leq g^{*}+e^{*} .
$$

Since $f$ and $g$ are convex, it follows

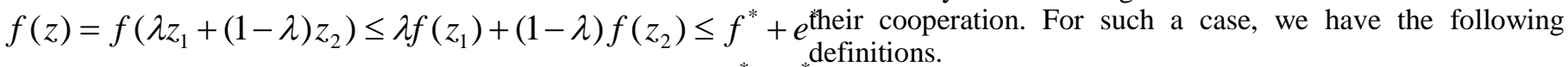
$g(z)=g\left(\lambda z_{1}+(1-\lambda) z_{2}\right) \leq \lambda g\left(z_{1}\right)+(1-\lambda) g\left(z_{2}\right) \leq g^{*}+e^{*}$ that is $f(z)-e^{*} \leq f^{*}, g(z)-e^{*} \leq g^{*}$, which implies $z$ is an $e^{*}$-optimal equilibrium solution of the supplier and the retailer. Thus $Z \in X^{*}$ and $X^{*}$ is convex. This completes the proof.

By Theorem (2), we have the following result.

Corollary (1). Suppose that $X$ is convex, and both $f(\cdot)$ and $g(\cdot)$ are strictly convex. Then the $e^{*}$-optimal equilibrium solution of the supplier and the retailer is unique.

Now, we will show how to find the $e^{*}$-optimal equilibrium solution of the supplier and the retailer. First, we will introduce the following mathematical programming problem (PE)

(PE) $\min e$

$$
\begin{aligned}
& \text { s.t. } f(x, y, u, v)-e \leq f^{*}, g(x, y, u, v)-e \leq g^{*}, \\
& (x, y, u, v) \in X .
\end{aligned}
$$

Then we have the following result.

Theorem (3). Suppose that $\left(\left(x^{*}, y^{*}, u^{*}, v^{*}\right), e^{*}\right)$ is an optimal solution of problem (PE). Then $\left(x^{*}, y^{*}, u^{*}, v^{*}\right)$ is an $e^{*}$-optimal equilibrium solution of the supplier and the retailer.

Proof. Suppose that $\left(\left(x^{*}, y^{*}, u^{*}, v^{*}\right), e^{*}\right)$ is an optimal solution of problem (PE). It follows with the constraint conditions of (PE) that $f\left(x^{*}, y^{*}, u^{*}, v^{*}\right)-e^{*} \leq f^{*}$,

$$
g\left(x^{*}, y^{*}, u^{*}, v^{*}\right)-e^{*} \leq g^{*}, \quad\left(x^{*}, y^{*}, u^{*}, v^{*}\right) \in X .
$$

Then it follows with definition (2) that $\left(x^{*}, y^{*}, u^{*}, v^{*}\right)$ is an $e^{*}$-equilibrium solution of the supplier and the retailer. Let $\left(x^{\prime}, y^{\prime}, u^{\prime}, v^{\prime}\right)$ be an $e^{\prime}-$ optimal equilibrium solution of the supplier and the retailer, then it follows with definition (3) that $e^{\prime} \leq e^{*}$. Besides, $\left(\left(x^{\prime}, y^{\prime}, u^{\prime}, v^{\prime}\right), e^{\prime}\right)$ is a feasible solution to problem (PE) since it satisfies the constraint conditions of problem (PE). This implies $e^{*} \leq e^{\prime}$ since $\left(\left(x^{*}, y^{*}, u^{*}, v^{*}\right), e^{*}\right)$ and $\left(x^{*}, y^{*}, u^{*}, v^{*}\right)$ turns to be an $e^{*}$-optimal equilibrium solution of the supplier and the retailer. This completes the proof.

By Theorem (3), we can obtain the $e^{*}$-optimal equilibrium solution of the supplier and the retailer by solving the corresponding problem (PE). In reality, the supplier and the retailer may be want to give different concession values for is an optimal solution of problem (PE). Then we have $e^{\prime}=e^{*}$ 
Definition (4). Let $f^{*}$ be the optimal joint value of the supplier and $g^{*}$ be the optimal joint value of the retailer. For $e \geq 0$ and $(x, y, u, v) \in X$, let $w=\left(w_{1}, w_{2}\right)>0$ be the concession weight of the supplier and the retailer. If it satisfies

$$
f(x, y, u, v)-w_{1} e \leq f^{*}, g(x, y, u, v)-w_{2} e \leq g^{*},
$$

$(x, y, u, v)$ is called an $e$ - equilibrium solution of the supplier and the retailer with respect to $w . e$ is called an equilibrium value of the supplier and the retailer with respect to $w$.The set of all the equilibrium values of the supplier and the retailer with respect to $w$ is denoted by $E_{w}$.

Definition (5). For the concession weight $w=\left(w_{1}, w_{2}\right)>0$, let $e^{*}$ be the minimum element in $E_{w}$ and $\left(x^{*}, y^{*}, u^{*}, v^{*}\right)$ be an $e^{*}-$ equilibrium solution of the supplier and the retailer with respect to $w$. Then $\left(x^{*}, y^{*}, u^{*}, v^{*}\right)$ is called an $e^{*}$-optimal equilibrium solution of the supplier and the retailer with respect to $w . e^{*}$ is called an optimal equilibrium value of the supplier and the retailer with respect to $w$.

For the CC model and $w=\left(w_{1}, w_{2}\right)>0$, we introduce the following corresponding $C C_{w}$ model as follows:

$$
\begin{aligned}
(\mathrm{PM}) \min f_{w}(x, y, u, v) & =\frac{1}{w_{1}} f(x, y, u, v) \\
& =\frac{1}{w_{1}}\left[u+\frac{1}{1-\beta} E\left[(L(x, y, \xi)-u)^{+}\right]\right]
\end{aligned}
$$

s.t. $r<x, b<x, x<a, u \in R$.

(PR) min $g_{w}(x, y, u, v)=\frac{1}{w_{2}} g(x, y, u, v)$

$=\frac{1}{w_{2}}\left[v+\frac{1}{1-\alpha} E\left[(l(x, y, \eta)-v)^{+}\right]\right]$s.t. $n \leq y \leq N, v \in R$.

Then we have the following result.

Theorem (4). For $w=\left(w_{1}, w_{2}\right)>0$, suppose that $(x, y, u, v)$ is an $e-$ equilibriumsolution of the $C C_{w}$ model. Then $(x, y, u, v)$ is an $e$-equilibrium solution of $C C$ model with respect to $w$.

Proof. Let $(x, y, u, v)$ be an e $e$-equilibrium solution of $C C_{w}$ model, it follows with definition (2) that

$$
\frac{1}{w_{1}} f(x, y, u, v)-e \leq \frac{1}{w_{1}} f^{*}, \frac{1}{w_{2}} g(x, y, u, v)-e \leq \frac{1}{w_{2}} g^{*},
$$

which implies

$$
f(x, y, u, v)-w_{1} e \leq f^{*}, g(x, y, u, v)-w_{2} e \leq g^{*} .
$$

Then $(x, y, u, v)$ turns to be an $e$-optimal equilibrium solution of $C C$ model with respect to $w$ by definition (4). This completes the proof.

By Theorem (4), we can obtain the $e$-equilibrium solution of the supplier and the retailer with respect to $w$ by computing the $e$-equilibrium solution of the corresponding $C C_{w}$ model. We define the mathematical programming problem $\left(P E_{w}\right)$ as follows :

$$
\begin{aligned}
& \left(P E_{q}\right) \min \quad e \\
& \text { s.t. } f(x, y, u, v)-w_{1} e \leq f^{*}, g(x, y, u, v)-w_{2} e \leq g^{*}, \\
& \quad(x, y, u, v) \in X
\end{aligned}
$$

Then the following result is achieved.

Theorem (5). For $w=\left(w_{1}, w_{2}\right)>0$, suppose that $\left(\left(x^{*}, y^{*}, u^{*}, v^{*}\right), e^{*}\right)$ is an optimal solution of problem $\left(P E_{w}\right)$. Then $\left(x^{*}, y^{*}, u^{*}, v^{*}\right)$ is an $e^{*}$-optimal equilibrium solution of the supplier and the retailer with respect to $w$.

For $w=\left(w_{1}, w_{2}\right)>0$, we can obtain the $e^{*}$-optimal equilibrium solution of the supplier and the retailer with respect to $w$ by solving the corresponding problem $\left(P E_{w}\right)$.

\section{NUMERICAL EXAMPLE}

In order to illustrate the efficiency of the $C C$ model proposed in Section III, we will give a numerical example and some sensitivity analysis.

Example (1): Considering the two-stage supply chain consisting of a supplier and a retailer. Suppose that the product's stochastic price $\xi$ subjects to the normal distribution $N\left(3,0.8^{2}\right)$ and the the product's stochastic demand $\eta$ subjects to the normal distribution $N\left(100,25^{2}\right)$. The corresponding parameters are given as follows: $b=1, a=5, n$ $=80, \mathrm{~N}=120$. For $\mathrm{r}=0.5, \mathrm{~s}=1$ and $\alpha=\beta=0.80$, let $\mathrm{t}=0.1$, $\mathrm{t}=0.3$ and $\mathrm{t}=0.5$, respectively. The results from the CC model under the above conditions are listed in Table I.

TABLE I. RESULTS FROM THE CC MODEL WITH $\mathrm{R}=0.5, \mathrm{~S}=1$ AND A $=\mathrm{B}=0.80$

\begin{tabular}{|c|c|c|c|c|c|c|c|}
\hline $\mathbf{t}$ & $\mathbf{x}$ & $\mathbf{y}$ & $f^{*}$ & $g^{*}$ & $\pi_{1}$ & $\pi_{2}$ & $e^{*}$ \\
\hline 0 & 2.68 & 80 & 9.09 & 16.27 & 39.93 & 47.11 & 30.84 \\
\hline 0.3 & 2.54 & 80 & 23.08 & 16.27 & 50.94 & 44.13 & 27.86 \\
\hline 0.5 & 2.44 & 80 & 33.33 & 16.27 & 59.01 & 41.95 & 25.68 \\
\hline
\end{tabular}

By Table I, it is easily found that when the supplier's excess penalty coefficient $t$ increases, the supplier's wholesale price $x$ will decrease, and the optimal equilibrium value $e^{*}$ will decrease, too. If $t$ increases, the supplier's loss will increase when he gives a high wholesale price. Then the supplier should 
better give a smaller wholesale price to ensure the future cooperation with the retailer.

For $\mathrm{t}=0.3, \mathrm{r}=0.5$ and $\mathrm{s}=1$, let $a=\beta=0.80, \alpha=\beta=$ 0.85 and $\alpha=\beta=0.90$, respectively. The results from the CC model under the above conditions are listed in Table II.

TABLE II. RESULTS OF THE EXAMPLE WITH $\mathrm{T}=0.3, \mathrm{R}=0.5$, AND $\mathrm{S}=1$

\begin{tabular}{|c|c|c|c|c|c|c|c|c|}
\hline$\alpha$ & $\beta$ & $x$ & $y$ & $f^{*}$ & $g^{*}$ & $\pi_{1}$ & $\pi_{2}$ & $e^{*}$ \\
\hline 0.80 & 0.80 & 2.54 & 80 & 23.08 & 16.27 & 50.94 & 44.13 & 27.86 \\
\hline 0.85 & 0.85 & 2.52 & 80 & 28.85 & 20.33 & 52.29 & 43.77 & 23.44 \\
\hline 0.90 & 0.90 & 2.49 & 80 & 38.54 & 27.17 & 54.53 & 43.16 & 15.99 \\
\hline
\end{tabular}

By Table II, it is easily found that when the confidence levels $\alpha$ and $\beta$ increase, the supplier's wholesale price will decrease and the optimal equilibrium value $e^{*}$ will decrease, too. The confidence levels $\alpha$ and $\beta$ indicate the risk preferences of the supplier and the retailer, respectively. When $\alpha$ and $\beta$ grow bigger, the supplier and the retailer becomes more risk-averse. In such a case, the supplier should give a smaller wholesale price and the optimal equilibrium value $e^{*}$ will decrease.

For $\mathrm{t}=0.3, \mathrm{r}=0.5, \mathrm{~s}=1$ and $\alpha=\beta=0.80$, let $w=\left(w_{1}, w_{2}\right)=(0.67,0.33), w=\left(w_{1}, w_{2}\right)=(0.75,0.25)$ and $w=\left(w_{1}, w_{2}\right)=(0.80,0.20)$, respectively. The results from the CC model under the above conditions are listed in Table III.

TABLE III. RESULTS OF THE EXAMPLE WITH $\mathrm{R}=0.5, \mathrm{~S}=1$ AND $\mathrm{A}=\mathrm{B}=0.80$

\begin{tabular}{|c|c|c|c|c|c|c|c|}
\hline$\left(w_{1}, w_{2}\right)$ & $x$ & $y$ & $f^{*}$ & $g^{*}$ & $\pi_{1}$ & $\pi_{2}$ & $e^{*}$ \\
\hline$(0.67,0.33)$ & 1.61 & 81.51 & 23.08 & 16.27 & 42.10 & 25.78 & 28.53 \\
\hline$(0.75,0.25)$ & 1.48 & 83.07 & 23.08 & 16.27 & 44.04 & 26.75 & 31.44 \\
\hline$(0.80,0.20)$ & 1.39 & 84.25 & 23.08 & 16.27 & 45.36 & 27.41 & 33.42 \\
\hline
\end{tabular}

By Table III, it is found that when the value of $\frac{w_{1}}{w_{2}}$ increases, which implies the supplier gives more concessions in the cooperation, the supplier's wholesale price will decrease. For such a case, the retailer's order quantity will increase and the optimal equilibrium value $e^{*}$ will increase, too.

To summarize this section, we can conclude that the proposed method is efficient to enhance the cooperation between the supplier and the retailer. Both the supplier and the retailer will do their best to cooperate with each other. The $e^{*}-$ optimal equilibrium solution provides a fair solution to the supplier and the retailer and can ensure the minimum concession in their cooperation.

\section{CONCLUSIONS}

In this paper, we introduce a Cooperation and Competition model for the risk management of the two-stage supply chain consisting of a supplier and a retailer. This model considers the risk aversion of the supplier and the retailer by introducing the CVaR objective. The $e^{*}$-optimal equilibrium solution to this model can coordinate the decisions of the supplier and the retailer and enhance their cooperations. Thus this paper also contributes to the risk management of the two-stage supply chain.

\section{ACKNOWLEDGMENT}

This research is supported by Zhejiang Provincial Natural Science Foundation of China under Grant No.LY 13G020028 and No.LY15G010007.

\section{REFERENCES}

[1] Simon Croom, Pietro Romano, Mihalis Giannakis, Supply chain management: an analytical framework for critical literature review, European Journal of Purchasing \& Supply Management, 2000, 6: 67-83.

[2] Christopher S. Tang, Perspectives in supply chain risk management, International Journal of Production Economics, 2006, 103: 451-488.

[3] Mark Goh, Joseph Y.S. Lim, Fanwei Meng, A stochastic model for risk management in global supply chain networks, European Journal of Operational Research, 2007,182: 164-173.

[4] R.T. Rockafellar, Stanislav Uryasev, Optimization of Conditional Valueat-Risk, The Journal of Risk, 2000, 2: 21-41.

[5] R.T. Rockafellar, Stanislav Uryasev, Conditional value-at-risk for general loss distributions, Journal of Banking \& Finance, 2002, 26: 1443-1471.

[6] Chen X., Sim M., Simichi-levi D, Sun P, Risk Aversion in Inventory Management, MIT, Cambridge, MA, 2003.

[7] Xu Minghui, Yu Gang, Zhang Hanqin, CVaR in a newsvendor model with lost sale penalty cost(in Chinese), Systems Engineering: Theory and Application, 2006, 10:1-8.

[8] Juan-ya Gotoh, Yuichi Takano, Newsvendor solutions via conditional value-at-risk minimization, European Journal of operational research, 2007, 179: 80-96.

[9] Lu Cheng, Zhongping Wan, Guangmin Wang, Bilevel newsvendor models considering retailer with CVaR objective, Computers \& Industrial Engineering, 2009, 57:310-318.

[10] Chung-Chi Hsieh, Yu-Ting Lu, Manufacturer, return policy in a twostage supply chain with two risk-averse retailers and random demand, European Journal of Operational Research, 2010, 207: 514-523.

[11] Ozgun Caliskan-Demirag, Youhua Chen, Jianbin Li, Customer and Retailer rebates under risk aversion, International Journal of Production Economics, 2011, 133: 736-750.

[12] Artzner P., Delbaen F., J. M. Eber, D. Heath, Coherent measures of Risk, Mathematical Finance, 1999, 9: 203-228.

[13] Mauser H., Rosen D., Beyond VaR: From measuring risk to managing risk, ALGO Research Quarterly, 1999, 1: 5-20. 